\title{
ARTICLE
}

\section{Body mass index and common mental disorders: exploring the shape of the association and its moderation by age, gender and education}

\section{Running title: BMI and common mental disorders}

Rachel L McCrea MSc

Department of Mental Health Sciences, University College London, UK

Yves G Berger PhD

Social Statistics, University of Southampton, UK

Michael B King MD PhD

Department of Mental Health Sciences, University College London, UK

The published version of this paper is available at:

McCrea, R.L., Berger, Y.G., King, M.B., 2012. Body mass index and common mental disorders: exploring the shape of the association and its moderation by age, gender and education. International Journal of Obesity 36, 414-421. doi:10.1038/ijo.2011.65

http://www.nature.com/ijo/journal/v36/n3/abs/ijo201165a.html 


\section{Abstract}

Background: Obesity is known to be associated with increased prevalence of common mental disorders (e.g. depression and anxiety), and there is evidence of age and gender differences in this relationship. However, categorisation of body mass index (BMI) and age has limited our ability to understand the nature of these differences. This study used continuous values of BMI and age to explore the shape of the association between common mental disorders and BMI and whether it varied with age, gender and education.

Method: The analysis used cross-sectional data on 7043 adults from the English 2007 Adult Psychiatric Morbidity Survey. Common mental disorders were assessed using the revised Clinical Interview Schedule (CIS-R). Cubic splines allowed BMI and age to have non-linear effects in the logistic regression analysis.

Results: BMI was strongly associated with the presence of common mental disorders, and there was clear evidence that this association varied with gender and age. In young women the probability of having a disorder increased as BMI increased, while in young men the relationship was $U$-shaped - probabilities were higher for both underweight and obese men. These associations diminished in older age groups, particularly when potential confounders such as physical health were taken into account. There was no evidence that the relationship varied with education.

Conclusions: Age and gender differences must be taken into account when investigating the link between BMI or obesity and common mental disorders. Furthermore, results of studies that categorise BMI may be highly sensitive to the width of the 'normal weight' reference category. 
Keywords: depression; anxiety; common mental disorders; body mass index 


\section{Introduction}

Obesity and its relationship with common mental disorders (such as depression and anxiety) has been the focus of much research in recent years. At least 26 cross-sectional studies have investigated the association between obesity or body mass index (BMI) and depression in adult community samples, ${ }^{1,2}$ and a recent meta-analysis found that obese individuals were 1.18 times more likely to be depressed than those below the obesity threshold $(\mathrm{BMI}>=30)$. Similar results have been found in studies investigating the relationship between obesity and anxiety disorders,,${ }^{3,4}$ and also in a recent meta-analysis of longitudinal studies investigating the association between obesity and depression. ${ }^{5}$

A key question in this research is the nature of age and gender differences in the relationship - several studies have found such differences, ${ }^{6-9}$ but their directions have not always been consistent. For example, one study found that in men obesity but not being overweight (BMI $25-<30$ ) increased the odds of having depression, whereas in women both obesity and being overweight increased the odds of having the condition. ${ }^{7}$ However, another study found the converse - being overweight was associated with increased depression in men (in their adjusted analysis only), but not among women. ${ }^{9}$ With regard to age differences, while one study found no significant effects of age, ${ }^{3}$ another study found clear age differences, with much less evidence for the association in those over 65 than in younger individuals. ${ }^{8}$

It can be challenging to investigate such age and gender differences with even very large datasets - the cross-classification of BMI categories by age group and gender (coupled [Page $415 \rightarrow$ ] with a low point prevalence of anxiety or depressive disorders) can result in small cells with few or no cases of disorders, unstable estimates and very wide confidence intervals. This may explain why previous studies have shown inconsistent results in the differences they report. The present study aims to elucidate age and gender differences in 
the association between $\mathrm{BMI}$ and common mental disorders by using a statistical technique that allows continuous variables to have non-linear relationships with the outcome variable. By avoiding the categorisation of $\mathrm{BMI}$ and age, full use is made of all information in the data and the directions of age and gender differences are clarified. The study will also check for any differences related to highest educational qualification. The analysis uses data from an English population survey, and its key focus will be on describing the shape of the relationship with $\mathrm{BMI}$, and how this varies in the different age, gender and education groups.

\section{Methods}

\section{Source}

The data for this study come from the 2007 Adult Psychiatric Morbidity Survey, the most recent of three surveys investigating the mental health of the UK population. The 2007 survey was a cross-sectional household survey of adults aged 16 or over living in England. It was carried out by the National Centre for Social Research and was sponsored by the NHS Information Centre for Health and Social Care. The dataset was supplied by the UK

Data Archive. ${ }^{10} \mathrm{~A}$ detailed account of the survey method is available elsewhere. ${ }^{11}$ The survey used a multi-stage probability sample based on the small users' Postcode Address File - 519 English postal sectors, stratified by region and socio-economic characteristics, were selected with inclusion probability proportional to size. 28 addresses were selected at random within each postal sector. Trained interviewers visited each selected address and selected one adult at random to be interviewed from each eligible household (in multihousehold addresses, a single household was selected at random within the address). Interviews were carried out with computer-assisted personal interviewing (CAPI) and lasted on average approximately one and a half hours. The sample was designed to be representative of the English adult household population, although there was some 
underrepresentation of young adults (particularly men), as a result of differential response rates between age groups. ${ }^{11}$ The overall response rate for the survey was 57\% - 7403 individuals completed at least the clinical interview section of the questionnaire. A summary of the demographic characteristics of the study sample can be seen in Table 1 .

\section{Measures}

Common mental disorders were measured using the revised Clinical Interview Schedule $(\mathrm{CIS}-\mathrm{R})$ that focuses on [Page $\mathbf{4 1 6} \rightarrow$ ] non-psychotic symptoms experienced in the week before the interview. ${ }^{12} \mathrm{~A}$ set of algorithms ${ }^{13}$ was used to obtain diagnoses of 13 disorders based on ICD-10 diagnostic criteria. ${ }^{14}$ The diagnoses were grouped into six categories of disorders - depressive episodes, generalised anxiety disorder, phobias, obsessive compulsive disorder, panic disorder and mixed anxiety and depressive disorder. The diagnosis of mixed anxiety and depressive disorder was given to those with a CIS-R score of 12 or more (the standard cut-off indicating a psychiatric case) ${ }^{12}$ but not meeting the diagnostic criteria for any other common mental disorder covered by the CIS-R. For most of this study all six categories of disorder described above were combined into a single outcome of 'any common mental disorder', in order to ensure a sufficient number of 'events per variable' for logistic regression with non-linear terms and complex high-order interactions. $^{15}$

BMI was calculated as weight (in kilograms) divided by height (in metres) squared, and was derived from self-reported weight and height recorded in the interview. Where BMI was categorised, this was according to the $\mathrm{WHO}$ International Classification that defines $\mathrm{BMI}<18.5$ as 'underweight', 18.5 to $<25$ as 'normal weight', 25 to $<30$ as 'overweight', 30 to $<35$ as 'obese class I', 35 to $<40$ as 'obese class II' and $>=40$ as 'obese class III'. ${ }^{16} 77$ women who were pregnant at the time of interview were excluded from the study sample. 
Other variables in the analyses were respondents' gender, age (respondents aged 95 or over were merged at 95 in the archived dataset to protect confidentiality), highest educational qualification already achieved (university level, secondary school level and none), ethnicity (white, black, South Asian and other/'don't know'), employment status (employed, unemployed and economically inactive), marital status (married, widowed, divorced/separated and single/cohabiting), smoking status (current smoker, former smoker and never smoked), alcohol dependence and hazardous alcohol use, number of adults in the household, and number of chronic physical health conditions. Number of physical health conditions was a count of the diagnosed conditions that respondents identified from the following list: cancer, diabetes, epilepsy/fits, migraine or frequent headaches, dementia or Alzheimer's disease, cataracts/eyesight problems, ear/hearing problems, stroke, heart attack/angina, high blood pressure, bronchitis/emphysema, asthma, allergies, stomach ulcer or other digestive problems, liver problems, bowel/colon problems, bladder problems/incontinence, arthritis, bone, back, joint or muscle problems, infectious disease or skin problems. Conditions were only included if they had lasted for 12 months or more. Hazardous alcohol use was identified as a score of 8 or more on the Alcohol Use Disorders Identification Test (AUDIT). ${ }^{17}$ Those scoring 10 or more were asked to complete the Severity of Alcohol Dependence Questionnaire - Community Version (SADQ-C). ${ }^{18} \mathrm{~A}$ score of 4 or more on this measure was considered as alcohol dependence.

\section{Statistical analyses}

Prevalence estimates were calculated using the survey ('svy') commands in Stata for Windows (Version 10.1; StataCorp, College Station, TX, USA) to account for the stratified and clustered design. All prevalence estimates are weighted to account for unequal probabilities of selection (inversely related to household size) and differential nonresponse. 
Logistic regression was used to investigate the association between $\mathrm{BMI}$ and the presence of any common mental disorder. The variables and interactions to be included in the model were pre-specified following a review of the literature - no data-driven model selection procedures were used. Two models were fitted: the first contained BMI, gender, age, education and number of adults in the household as explanatory variables (number in the household was included because it affected the probability of inclusion in the sample); the second model additionally contained variables considered to be potential confounders (ethnicity, employment status, marital status, smoking status, alcohol dependence/hazardous drinking and number of chronic physical health conditions). Since a model-based approach was used for regression analysis of the survey data, survey weights were not used. ${ }^{19}$

All continuous variables were fitted with restricted cubic splines that allowed their relationships with the outcome variable to be non-linear. The number of knots for each variable was decided before analysis began. It was initially intended to model BMI using 5 knots, in order to give sufficient flexibility to fit a U-shaped but irregular relationship with the presence of a mental disorder. However, this was reduced to 4 knots because of estimation difficulties relating to the interaction with age. All other continuous variables were modelled with 3 knots (since there was less reason to expect that these variables would show such complex relationships with the outcome variable). Knots were placed at their default locations - for BMI, these were at the $5^{\text {th }}, 35^{\text {th }}, 65^{\text {th }}$ and $95^{\text {th }}$ percentiles of the sample BMI distribution (this resulted in knots at BMI values of 19.6, 23.9, 27.1 and 35.3). For other variables, the default knot locations were at the $10^{\text {th }}, 50^{\text {th }}$ and $90^{\text {th }}$ percentiles of their sample distributions. Both models included the three-way interaction terms BMl×gender $\times$ age and BMl×gender×education - these prevented age or education differences from being constrained to be the same for both genders. Other explanatory variables were also allowed to interact with gender (apart from the three variables with one 
or more very small categories - ethnicity, employment status and harmful drinking/alcohol dependence).

Logistic regression modelling was carried out in $\mathrm{R}$ for Windows (Version 2.11.0; www.rproject.org) with the Design library, ${ }^{20}$ incorporating Huber-White robust covariance estimates to account for the clustering of the sample. 283 participants had missing values for BMI and are excluded from all analyses and tables. In addition, 161 individuals had missing values for highest educational qualification and a further 13 individuals had missing values [Page $\mathbf{4 1 7} \rightarrow$ ] on one or more of the potential confounder variables - they were excluded from models including those variables. After model fitting, both models were examined for influential points using DFBETAS and checked for sensitivity to these influential points.

Key interaction terms in the model were evaluated using Wald tests to test whether the relevant parameters in the logistic regression model all equalled 0 . Logistic regression models incorporating many interactions terms and cubic splines produce parameter estimates that are extremely difficult to interpret. ${ }^{15}$ Therefore, predicted probability plots are used to explain the model results - any variables not specified in chart axes or legends were fixed at their median (for continuous variables) or mode (for categorical variables). These charts are plotted for BMI values between 17.5 and 44 (the $1^{\text {st }}$ and $99^{\text {th }}$ percentiles of the sample BMI distribution).

\section{Results}

\section{Descriptive statistics}

The characteristics of the 7043 individuals in the study sample with a valid BMI are shown in Table 1. BMI ranged from 12.5 to 81.4. The mean BMI of the sample was 26.2 (s.d. 5.4) while the median was 25.4. The total number of cases of common mental disorders was 1 
210. The estimated (weighted) prevalence of common mental disorders in the week prior to interview was $16.2 \%$ (Cl: $15.2-17.3 \%$ ). Prevalences of the six categories of ICD-10 diagnoses were as follows: depressive episodes: $2.3 \%(\mathrm{Cl}: 1.9-2.7 \%)$; panic disorder: 1.1\% (Cl: $0.8-1.4 \%)$; generalised anxiety disorder: $4.5 \%$ (Cl: $3.9-5.0 \%)$; obsessive compulsive disorder: 1.1\% (Cl: $0.8-1.4 \%)$; phobias: $1.8 \%(\mathrm{Cl}: 1.4-2.1 \%)$; mixed anxiety and depressive disorder: $8.9 \%$ (Cl: $8.1-9.7 \%)$.

Estimates of the prevalence of common mental disorders for age and gender specific groups are shown in Figure 1. The wide $95 \%$ confidence intervals for the $\mathrm{BMI}<18.5$ and $\mathrm{BMI}>40$ categories reflect the small numbers of individuals in these groups. For women aged 16-59, the prevalence of common mental disorders is significantly higher in the $25-30$ and 30-35 BMl categories than in the normal weight category (BMI of 18.5-25). However, for women aged 60 and over the prevalence estimates are similar in these three categories. For men, most of the confidence intervals overlap, so it is difficult to draw conclusions about differences between the BMI categories. However, for men aged 16-39 the prevalence of common mental disorders is significantly greater in the BMI $40+$ category than in the $25-<30$ category.

\section{Results of key hypothesis tests}

The results from our two logistic models were similar ([Page $418 \rightarrow$ ]

Table 2) and therefore most of the subsequent presentation will focus on the model including the potential confounding variables. BMI was strongly associated with the presence of a common mental disorder $(p=0.002)$. Tests for the BMl $\times$ gender and BMl×age interaction terms in the models suggest that the relationship between $\mathrm{BMI}$ and common mental disorders varies with age $(p=0.02)$ and gender $(p=0.15)$. The relationship between $\mathrm{BMI}$ and common mental disorders did not vary with education $(p=$ 
0.88). In the model without potential confounders there was less evidence for variation with age $(p=0.15)$, but stronger evidence for an association with gender $(p=0.03)$.

\section{Model interpretation}

We focus on predictions and odds ratios between the $5^{\text {th }}$ and $95^{\text {th }}$ percentiles of the sample BMI distribution (approximately BMI values of 20 and 35). Restricted cubic splines constrain the relationship with BMI to be a straight line beyond these [Page $418 \rightarrow$ ] points - consequently, the models may describe poorly some aspects of the relationship at the extremes of the BMI range. The predicted probabilities of having a common mental disorder for men and women aged 30 are shown in Figure 2. At this age, there is a striking gender difference in the shape of the association between $\mathrm{BMI}$ and common mental disorders. For men, the predicted probabilities follow a U-shape. The lowest probability of having a common mental disorder is 0.09 for those with a BMI of 24 or 25 , compared to 0.14 for an individual with the same 'average' characteristics but a BMI of 20. Meanwhile, there is also an increase in predicted probabilities for those with a BMI greater than 25. (The wide 95\% confidence intervals for BMI values below 20 and above 35 reflect the small numbers on which these sections of the model are based.) For women aged 30 the predicted probabilities increase as BMI values increase from 20 to 30 - for a woman with 'average' characteristics, the probability of having a common mental disorder at a BMI of 20 is 0.13 , compared to 0.23 at a BMI of 30 .

Age differences in the fitted relationship between BMl and the probability of having a common mental disorder are shown in Figure 3. For men, the rise in predicted probabilities of having a common mental disorder at low BMI values remains until about age 50 . At the other end of the scale, the increase in probabilities with high BMI values flattens out from around age 40 . For men in their 60 s and 70 s, the confidence intervals (not shown) are wide, relative to the observed variations in predicted probabilities. Nonetheless, the 
probability of having a common mental disorder appears to be lower at BMI values of 30 or over than at a BMI of around 25. For women, the association between BMI and the [Page $419 \rightarrow$ ] probability of having a common mental disorder starts to flatten out from the age of 40. Over the age of 60 , there is little evidence for any association between BMI and the probability of having a common mental disorder. Little can be concluded from the drop in probability for women aged 20 with very high BMI because there were so few women in the sample below the age of 25 with severe obesity - hence, the confidence intervals here are very wide.

While the complex logistic regression models used in this study do not provide regression parameter estimates that can be easily converted into odds ratios, odds ratios (relative to a chosen BMI value) can be calculated by the analysis software and plotted or tabulated - a selection are presented in [Page $419 \rightarrow]$

Table 3 and Figure 4 . The odds ratios shown here are relative to a BMI value of 25 (approximately the median of the study sample). Two particular odds ratios in [Page 419 $\rightarrow]$

Table 3 highlight the gender difference in the fitted model: for a woman aged 30 with a secondary qualification, a BMI of 20 is associated with 0.7 times lower odds of having a common mental disorder than a BMI of 25 ; however, for a man aged 30 , the same BMI difference is associated with 1.7 times greater odds.

Some of the differences between the models with and without potential confounders can be seen in Figure 4, which shows odds ratios from both models (relative to a BMI of 25) at ages 30 and 60 . The two models suggest similar shapes for the relationship between BMI and common mental disorders at age 30 . However, at age 60 there are more substantial differences between the two models. For men with a BMI between 30 and 35 there is more of a reduction in the odds ratio of having a common mental disorder in the model with 
confounders. For women aged 60 , in the model without confounders the increased odds ratios for high $\mathrm{BMI}$ values seen at younger ages remain, while in the model with confounders there is now little evidence for an association between BMI and the probability of having a common mental disorder. Therefore, the role of the potential confounders seems more important in older than in younger individuals.

\section{Discussion}

Adults in England show clear age and gender differences in the association between BMI and common mental disorders. For young men, there appears to be a U-shaped relationship between $\mathrm{BMI}$ and the probability of having a common mental disorder, whereas for young women higher BMI values are associated with a raised probability of having a common mental disorder. However, these relationships diminish in middle-aged and older individuals, (for women, this is particularly noticeable when potential confounders are taken into account). This variation with age may reflect age-related changes or cohort differences. The models fitted in this study provide no evidence for education differences [Page $\mathbf{4 2 0} \rightarrow$ ] in the relationship between BMI and the presence of common mental disorders.

\section{Comparison with previous studies}

The gender differences in the association between BMI and common mental disorders found in this study are consistent with other studies that have found a significant interaction between BMI category and gender. ${ }^{6,8,21,22}$ For example, a US study found that women who were overweight or obese had significantly increased odds ratios for suffering a mood or anxiety disorder, whereas for men this relationship held only for those who were obese (the odds of having a mood disorder were actually lower for overweight men than for those in the normal weight reference category) ${ }^{21}$ Few studies have looked specifically 
at age differences in the association between BMI category and common mental disorders. However, some significant age differences have been reported. ${ }^{3,8,9}$ For example, a meta-analysis of results from countries taking part in the World Mental Health Surveys initiative found age differences in the association between obesity and anxiety (but not depression), with significant differences from the reference category (BMI of 18.530) only for the youngest (18-34) and oldest (65+) age groups. ${ }^{3}$ However, the wide reference category used in these surveys precludes direct comparisons with our results.

Not all studies that have looked for age or gender differences have found them - a US study found no significant gender differences, ${ }^{23}$ while both that study and a Canadian study found no significant interaction with age. ${ }^{22,23}$ However, these studies dichotomised or categorised $\mathrm{BMI}$, and this may have limited their power to detect important differences. ${ }^{24}$ The US study also reported a significant interaction between obesity and years of educational attainment, unlike the present study that found no evidence for differences with level of education. The use of a continuous measure of educational attainment may have provided more power for their comparison. However, no adjustments for demographic characteristics appear to have been made during the test for the interaction, so it is not clear whether this association would remain after accounting for characteristics such as age, gender and ethnicity, or after including age and gender interaction terms.

\section{Strengths and limitations}

This study is the first to examine the relationship between BMl and the prevalence of common mental disorders in a nationally-representative English sample. A key strength is that BMI was modelled non-linearly as a continuous variable. This avoids the loss of information that results when a continuous variable is divided into broad categories. Furthermore, the directions of the age and gender differences can be seen more clearly than in previous studies that have categorised BMI and age. 
The main limitation of this study is that the response rate for the 2007 Adult Psychiatric Morbidity Survey was only $57 \%$ - the inferences made in this study rely on the assumption that the association between BMI and common mental disorders is the same in responders and non-responders, but it is not possible to test the validity of this assumption. A further limitation is that BMI in this study was derived from self-reported height and weight, which are known to be subject to reporting error and bias. ${ }^{25}$ However, the rank correlations between self-reported BMI and measured BMI have been found to be high. $^{26}$

The present study combined a number of common mental disorders into a single outcome variable, but it is possible that different relationships hold for different disorders - this is an important question for future research. It would also be interesting to see whether similar relationships hold for dimensional measures of depression and anxiety.

\section{Implications and future directions}

While this study was unable to investigate directly the causal relationship between BMI and common mental disorders, its gender- and age-specific results may help the formulation of future research questions. For example, the gender differences observed among young adults may be related to different ideal body shapes - young women may favour slimness, whereas young men may wish to be muscular and well built. Research into gender differences in patterns of body shape dissatisfaction might explain some of the differences in the relationship between BMI and common mental disorders. Furthermore, the observed age differences in the role of the potential confounders suggest that there may be different causal mechanisms at work in the younger and older age groups. Among young adults, confounding variables such as chronic physical health problems appear to contribute little to the increased prevalence of common mental disorders seen among the overweight and obese. However, among older adults (women in particular) confounders 
such as poor physical health appear to be much more important. We are hesitant to speculate about clinical implications, due to the cross-sectional nature of these data. However, clinicians should be sensitive to the finding that markedly underweight and obese younger men seem to encounter the most psychological difficulty, whereas for younger women poor mental health is found progressively more often as weight increases.

The results of this paper highlight the limitations of studies that ignore interactions with age or gender when investigating the association between BMI and common mental disorders. Not only do such studies overlook important differences, but the relationships in some groups may weaken or even cancel out when combined. Researchers would be strongly advised to include age and gender interaction terms in their models (even where not significant) or, failing that, to check their results by fitting separate models to the different age and gender groups. Furthermore, [Page $421 \rightarrow$ ] these results suggest that the age range of the sample will also be important - studies including predominantly older adults may be less likely to detect a relationship than those with many younger adults. Finally, the discovery of a U-shaped association with BMI for young men implies that studies which categorise BMI may be highly sensitive to the width of the 'normal weight' reference category. Where the reference category is very wide, there may be considerable heterogeneity within the category, and this may obscure the size and direction of the real relationship.

In summary, this study found clear age and gender differences in the relationship between $\mathrm{BMI}$ and common mental disorders, along with considerable variation within the standard BMI categories. Studies hoping to investigate the direction of the causal relationship must consider these differences, or they risk coming up with 'averaged' findings that fail to describe the actual relationship in any age or gender group. 
Conflict of interest

None. 


\section{References}

1 Atlantis E, Baker M. Obesity effects on depression: Systematic review of epidemiological studies. Int J Obes 2008; 32: 881-91.

2 de Wit L, Luppino F, van Straten A, Penninx B, Zitman F, Cuijpers P. Depression and obesity: A meta-analysis of community-based studies. Psychiatry Res 2010; 178: 230-5.

3 Scott KM, Bruffaerts R, Simon GE, Alonso J, Angermeyer M, De Girolamo G, et al. Obesity and mental disorders in the general population: Results from the world mental health surveys. Int J Obes 2008; 32: 192-200.

4 Mather AA, Cox BJ, Enns MW, Sareen J. Associations of obesity with psychiatric disorders and suicidal behaviors in a nationally representative sample. J Psychosom Res 2009; 66: 277-85.

5 Luppino FS, de Wit LM, Bouvy PF, Stijnen T, Cuijpers P, Penninx BWJH, et al. Overweight, obesity, and depression: A systematic review and meta-analysis of longitudinal studies. Arch Gen Psychiatry 2010; 67: 220-9.

6 de Wit LM, van Straten A, van Herten M, Penninx BWJH, Cuijpers P. Depression and body mass index, a u-shaped association. BMC Public Health 2009; 9: 14.

7 Zhao G, Ford ES, Dhingra S, Li C, Strine TW, Mokdad AH. Depression and anxiety among US adults: Associations with body mass index. Int J Obes 2009; 33: 257-66.

8 Heo M, Pietrobelli A, Fontaine KR, Sirey JA, Faith MS. Depressive mood and obesity in US adults: Comparison and moderation by sex, age, and race. Int J Obes 2006; 30: 513-9. 
9 Chen Y, Jiang Y, Mao Y. Association between obesity and depression in Canadians. J Womens Health 2009; 18: 1687-92.

10 National Centre for Social Research and University of Leicester. Adult Psychiatric Morbidity Survey, 2007 [Data File]. 2nd Edition. Colchester, Essex: UK Data Archive [distributor], April 2010. SN: 6379.

11 National Centre for Social Research. Adult psychiatric morbidity in England, 2007: Results of a household survey. National Centre for Social Research: London, 2009. Available at URL http://www.ic.nhs.uk/statistics-and-data-collections/mentalhealth/mental-health-surveys/adult-psychiatric-morbidity-in-england-2007:-results-ofa-household-survey (Accessed on 24/6/2009).

12 Lewis G, Pelosi AJ, Araya R, Dunn G. Measuring psychiatric disorder in the community - a standardized assessment for use by lay interviewers. Psychol Med 1992; 22: 465-86.

13 National Centre for Social Research. Adult psychiatric morbidity in England, 2007: Results of a household survey, Appendices and Glossary. National Centre for Social Research: London, 2009. Available at URL http://www.ic.nhs.uk/statistics-and-datacollections/mental-health/mental-health-surveys/adult-psychiatric-morbidity-inengland-2007:-results-of-a-household-survey_ (Accessed on 24/6/2009).

14 World Health Organization. The ICD-10 classification of mental and behavioural disorders: diagnostic criteria for research. World Health Organization: Geneva, 1993.

15 Harrell FE. Regression modeling strategies: with applications to linear models, logistic regression, and survival analysis. Springer-Verlag: New York, 2001. 
16 World Health Organization. Obesity: Preventing and managing the global epidemic. World Health Organization: Geneva, 2000. Available at URL http://apps.who.int/bmi/index.jsp?introPage=intro_5.html_ (Accessed on 26/8/2009).

17 World Health Organization. AUDIT - The alcohol use disorders identification test: Guidelines for use in primary care (Second edition). World Health Organization: Geneva, 2001. Available at URL http://www.who.int/substance_abuse/publications/alcohol/en/index.html_ (Accessed on $28 / 8 / 2009)$.

18 Stockwell T, Sitharthan T, Mcgrath D, Lang E. The measurement of alcohol dependence and impaired control in community samples. Addiction 1994; 89: 167-74.

19 Korn EL, Graubard Bl. Analysis of large health surveys - Accounting for the sampling design. Journal of the Royal Statistical Society Series A-Statistics in Society 1995; 158: 263-95.

20 Alzola C, Harrell F. An introduction to S and the Hmisc and Design libraries. 2006. Available at URL http://biostat.mc.vanderbilt.edu/wiki/pub/Main/RS/sintro.pdf (Accessed on 30/8/2009).

21 Barry D, Pietrzak RH, Petry NM. Gender differences in associations between body mass index and DSM-IV mood and anxiety disorders: Results from the National Epidemiologic Survey on Alcohol and Related Conditions. Ann Epidemiol 2008; 18: 458-66.

22 McLaren L, Beck CA, Patten SB, Fick GH, Adair CE. The relationship between body mass index and mental health - A population-based study of the effects of the definition of mental health. Soc Psychiatry Psychiatr Epidemiol 2008; 43: 63-71. 
23 Simon GE, Von Korff M, Saunders K, Miglioretti DL, Crane PK, van Belle G, et al. Association between obesity and psychiatric disorders in the US adult population. Arch Gen Psychiatry 2006; 63: 824-30.

24 Royston P, Altman DG, Sauerbrei W. Dichotomizing continuous predictors in multiple regression: A bad idea. Stat Med 2006; 25: 127-41.

25 Gorber SC, Tremblay M, Moher D, Gorber B. A comparison of direct vs. self-report measures for assessing height, weight and body mass index: A systematic review. Obes Rev 2007; 8: 307-26.

26 Spencer EA, Appleby PN, Davey GK, Key TJ. Validity of self-reported height and weight in 4808 EPIC-Oxford participants. Public Health Nutr 2002; 5: 561-5. 


\section{Tables}

\section{[Page $415 \rightarrow$ ]}

Table 1: Weighted estimates of the prevalence of any common mental disorder by each variable included in the models

\begin{tabular}{|c|c|c|c|c|c|}
\hline & & $\begin{array}{c}\text { Prevalence } \\
\text { of CMD }\end{array}$ & & & $\begin{array}{c}\text { Prevalence } \\
\text { of CMD }\end{array}$ \\
\hline Characteristic & $\mathrm{n}$ & $\%$ (s.e.) & Characteristic & $\mathrm{n}$ & $\%$ (s.e.) \\
\hline \multicolumn{3}{|c|}{ BMI (modelled continuously) } & \multicolumn{3}{|l|}{ Gender } \\
\hline$<18.5$ & 161 & $21.1(3.8)$ & Male & 3116 & $12.6(0.7)$ \\
\hline $18.5-<25$ & 3096 & $14.8(0.8)$ & Female & 3927 & $19.9(0.7)$ \\
\hline $25-<30$ & 2500 & $15.3(0.8)$ & & & \\
\hline $30-<35$ & 897 & $19.1(1.3)$ & Highest qualification & & \\
\hline $35-<40$ & 259 & $20.4(2.4)$ & University level & 1836 & $12.6(0.8)$ \\
\hline \multirow[t]{2}{*}{$40+$} & 130 & $35.0(4.7)$ & Secondary level & 2900 & $17.0(0.8)$ \\
\hline & & & No qualifications & 2146 & $18.4(1.0)$ \\
\hline \multicolumn{6}{|c|}{ Age (modelled continuously) } \\
\hline $16-39$ & 2132 & $18.2(0.9)$ & Employment status & & \\
\hline $40-59$ & 2356 & $19.0(0.9)$ & Employed & 3822 & $13.8(0.6)$ \\
\hline \multirow{2}{*}{$60+$} & 2528 & $9.9(0.6)$ & Unemployed & 153 & $30.6(4.8)$ \\
\hline & & & Economically inactive & 3068 & $19.2(0.8)$ \\
\hline \multicolumn{6}{|l|}{ Ethnicity } \\
\hline White & 6484 & $15.8(0.5)$ & Smoking status & & \\
\hline Black & 174 & $20.2(3.0)$ & Never smoked & 2378 & $13.5(0.7)$ \\
\hline South Asian & 188 & $16.3(2.8)$ & Current smoker & 1597 & $24.7(1.2)$ \\
\hline Other/don't know & 188 & $23.3(3.5)$ & Former smoker & 3066 & $13.8(0.7)$ \\
\hline \multicolumn{3}{|l|}{ Marital status } & \multicolumn{3}{|l|}{ Drinking status } \\
\hline Married & 3367 & $13.2(0.6)$ & No hazardous use & 6023 & $15.1(0.5)$ \\
\hline Widowed & 902 & $16.1(1.5)$ & Hazardous use & 650 & $14.4(1.6)$ \\
\hline Divorced/separated & 857 & $26.3(1.7)$ & Alcohol dependence & 358 & $35.2(2.8)$ \\
\hline \multirow[t]{2}{*}{ Never married } & 1917 & $18.9(1.0)$ & & & \\
\hline & & & \multicolumn{3}{|c|}{ Chronic health conditions (modelled continuously) } \\
\hline \multicolumn{3}{|c|}{ Adults in household (modelled continuously) } & 0 & 2538 & $10.2(0.7)$ \\
\hline 1 & 2312 & $22.2(0.9)$ & 1 & 1940 & $16.0(1.0)$ \\
\hline 2 & 3714 & $14.8(0.6)$ & 2 & 1209 & $21.2(1.4)$ \\
\hline 3 & 711 & $16.5(1.5)$ & 3 & 710 & $19.2(1.6)$ \\
\hline $4+$ & 306 & $12.1(1.8)$ & $4+$ & 644 & $36.3(2.2)$ \\
\hline
\end{tabular}

Abbreviation: CMD, common mental disorders 


\section{[Page $418 \rightarrow$ ]}

Table 2: Results of key Wald tests from fitting the logistic regression models with and without potential confounders ${ }^{\mathrm{a}}$

\begin{tabular}{|c|c|c|c|c|c|}
\hline \multirow[b]{2}{*}{ Test } & \multirow[b]{2}{*}{ d.f. ${ }^{d}$} & \multicolumn{2}{|c|}{$\begin{array}{l}\text { Model } 1^{\mathrm{b}} \text { (without } \\
\text { confounders) }\end{array}$} & \multicolumn{2}{|c|}{$\begin{array}{l}\text { Model } 2^{c} \text { (with } \\
\text { confounders) }\end{array}$} \\
\hline & & $\chi^{2}$ & $\mathrm{p}$ & $\chi^{2}$ & $\mathrm{p}$ \\
\hline BMI overall (including interactions) & 30 & 81.0 & $<0.0001$ & 57.9 & 0.0016 \\
\hline BMI $\times$ Gender & 15 & 26.3 & 0.0349 & 20.7 & 0.1483 \\
\hline $\mathrm{BMI} \times \mathrm{Age}$ & 12 & 17.1 & 0.1477 & 23.9 & 0.0212 \\
\hline $\mathrm{BMI} \times$ Education & 12 & 8.8 & 0.7166 & 6.5 & 0.8885 \\
\hline BMI $\times$ Gender $\times$ Age & 6 & 6.9 & 0.3312 & 6.6 & 0.3567 \\
\hline BMI $\times$ Gender $\times$ Education & 6 & 3.4 & 0.7557 & 3.1 & 0.8026 \\
\hline Total model & $43 / 64$ & 335.1 & $<0.0001$ & 870.9 & $<0.0001$ \\
\hline
\end{tabular}

${ }^{a}$ Both models also included number of adults in the household. Potential confounders were: ethnicity, employment status, marital status, smoking, alcohol dependence or hazardous use, and number of chronic physical health conditions.

${ }^{\mathrm{b}}$ Model 1: $\mathrm{n}=6,882 .{ }^{\mathrm{c}}$ Model 2: $\mathrm{n}=6,869$.

${ }^{d}$ The degrees of freedom for each Wald test incorporate the degrees of freedom for the term specified plus the degrees of freedom for any higher order interactions incorporating this term that were included in the model. 


\section{[Page $419 \rightarrow$ ]}

Table 3: Illustrative odds ratios for the probability of having a common mental disorder at selected BMI values, relative to someone with a BMI of $25^{\mathrm{a}}$

\begin{tabular}{|c|c|c|c|c|c|}
\hline \multirow[b]{2}{*}{ BMI value } & \multicolumn{2}{|c|}{$\begin{array}{l}\text { Odds ratio of having a CMD } \\
\text { relative to BMI of } 25\end{array}$} & \multirow[b]{2}{*}{ BMI value } & \multicolumn{2}{|c|}{$\begin{array}{l}\text { Odds ratio of having a CMD } \\
\text { relative to } \mathrm{BMI} \text { of } 25\end{array}$} \\
\hline & Estimate & $(95 \% \mathrm{Cl})$ & & Estimate & $(95 \% \mathrm{Cl})$ \\
\hline \multicolumn{3}{|c|}{ Man aged 30 with a secondary qualification } & \multicolumn{3}{|c|}{ Woman aged 30 with a secondary qualification } \\
\hline 20 & 1.71 & $(1.02,2.88)$ & 20 & 0.70 & $(0.51,0.98)$ \\
\hline 25 & 1.00 & 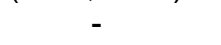 & 25 & 1.00 & \\
\hline 30 & 1.54 & $(1.01,2.33)$ & 30 & 1.38 & $(0.99,1.93)$ \\
\hline 35 & 1.61 & $(0.94,2.73)$ & 35 & 1.40 & $(0.97,2.01)$ \\
\hline \multicolumn{3}{|c|}{ Man aged 60 with a secondary qualification } & \multicolumn{3}{|c|}{ Woman aged 60 with a secondary qualification } \\
\hline 20 & 1.04 & $(0.50,2.18)$ & 20 & 0.95 & $(0.63,1.44)$ \\
\hline 25 & 1.00 & & 25 & 1.00 & \\
\hline 30 & 0.71 & $(0.44,1.16)$ & 30 & 1.03 & $(0.71,1.48)$ \\
\hline 35 & 0.73 & $(0.43,1.26)$ & 35 & 1.06 & $(0.70,1.59)$ \\
\hline
\end{tabular}

Abbreviation: CMD, common mental disorder

${ }^{a}$ Odds ratios are calculated from model 2 including potential confounders. 


\section{Figure legends}

\section{[Page $417 \rightarrow$ ]}

a)

b)
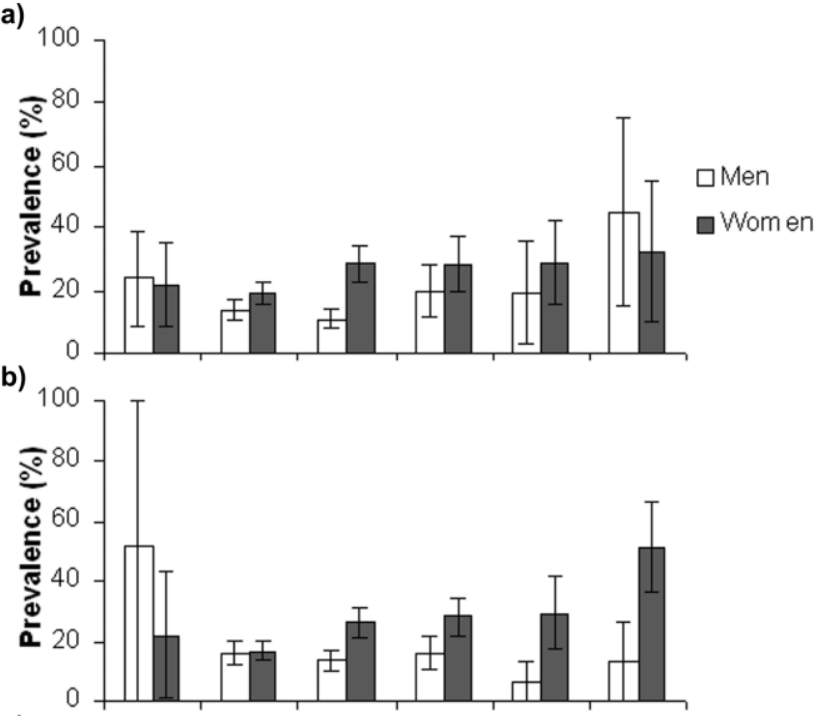

c)

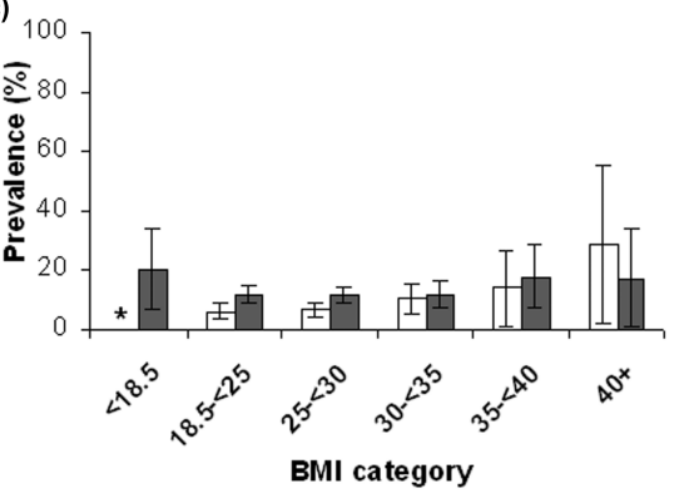

Figure 1: Prevalence of any common mental disorder with $95 \%$ confidence intervals by BMl category a) ages 16-39 b) ages 40-59 c) ages 60 and over. * No cases of common mental disorder in this category $(n=20)$. 
Men aged 30

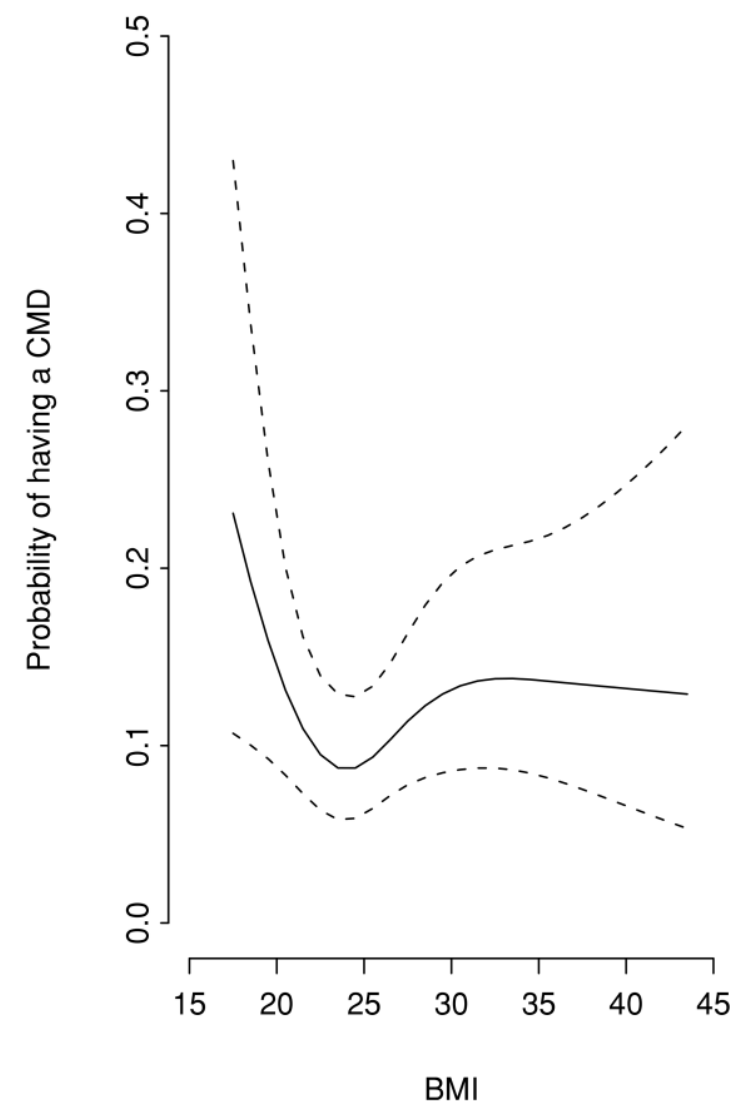

Women aged 30

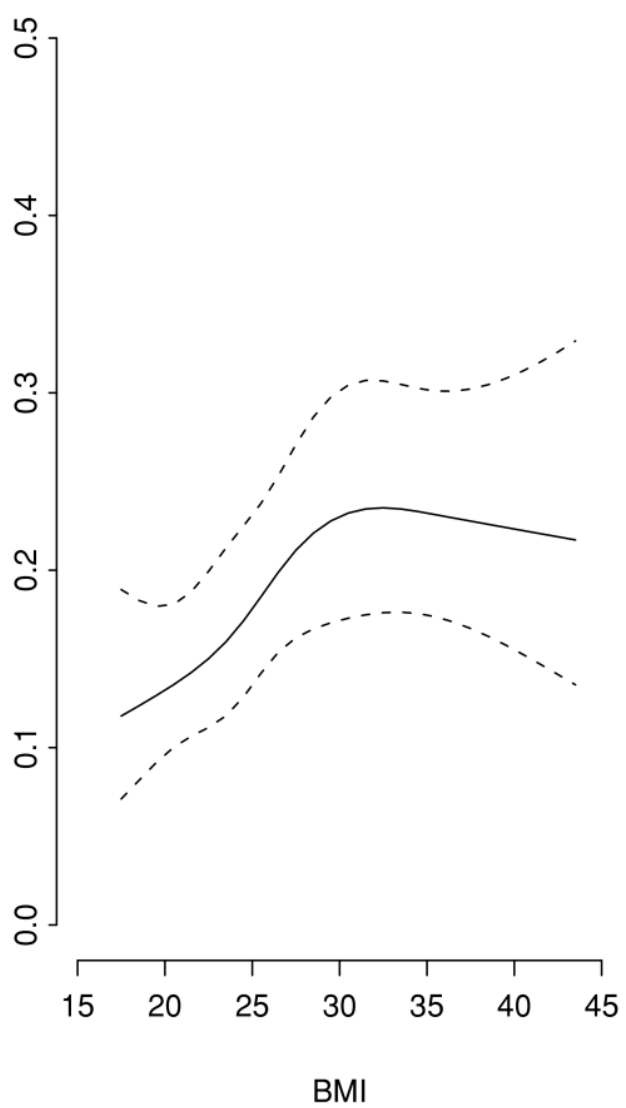

Figure 2: Predicted probabilities of having a common mental disorder at age 30 with $95 \%$ confidence intervals. All variables other than BMI, age and gender are held at fixed values. Categorical variables are fixed at their mode (see Table 1). Continuous variables are fixed at their median: number of physical health conditions $=1$; number of adults in household $=$ 2. 


\section{[Page $418 \rightarrow$ ]}

Men

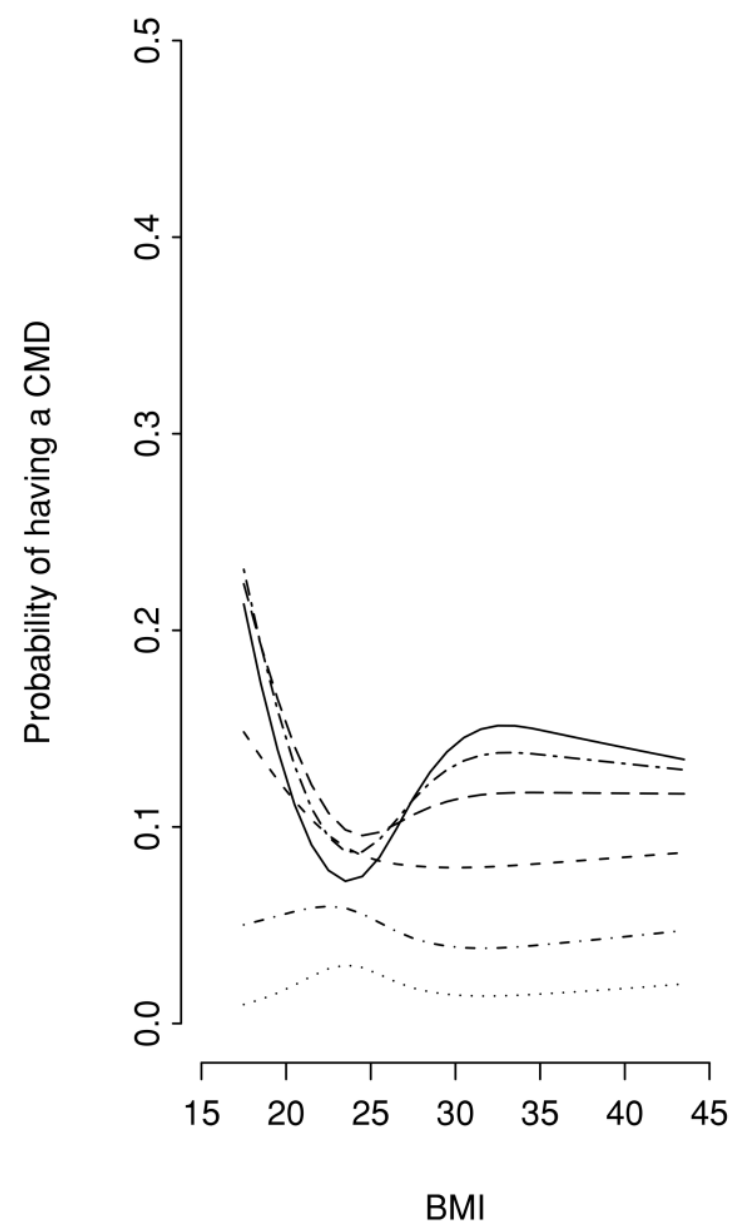

Women
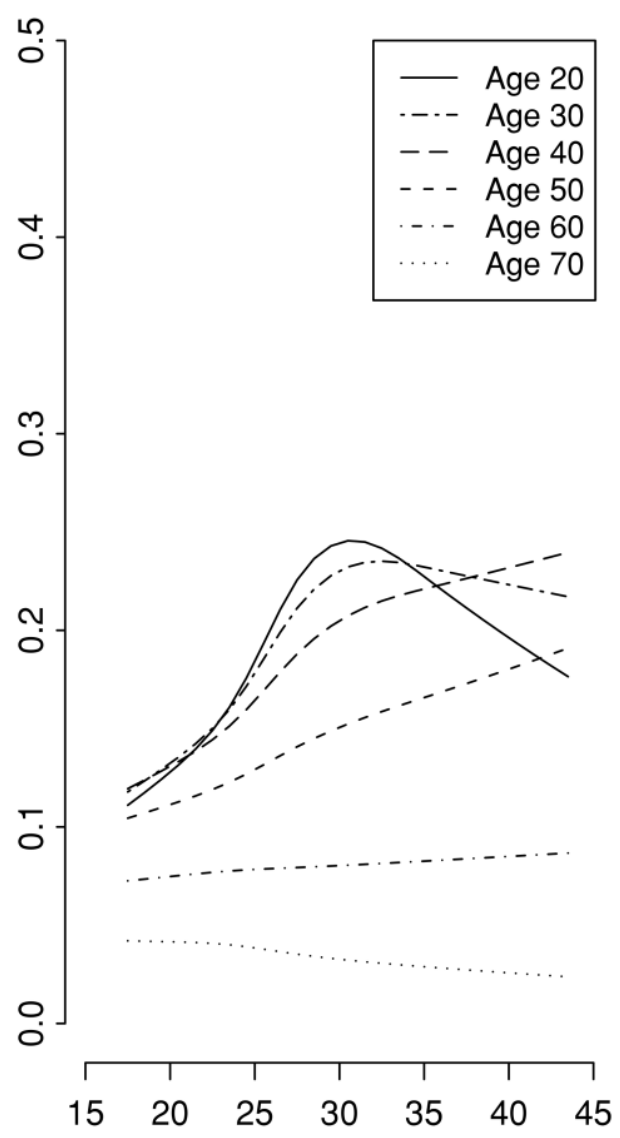

BMI

Figure 3: Predicted probabilities of having a common mental disorder at different ages. All variables other than $\mathrm{BMI}$, age and gender are held at fixed values. Categorical variables are fixed at their mode (see Table 1). Continuous variables are fixed at their median: number of physical health conditions $=1$; number of adults in household $=2$. 


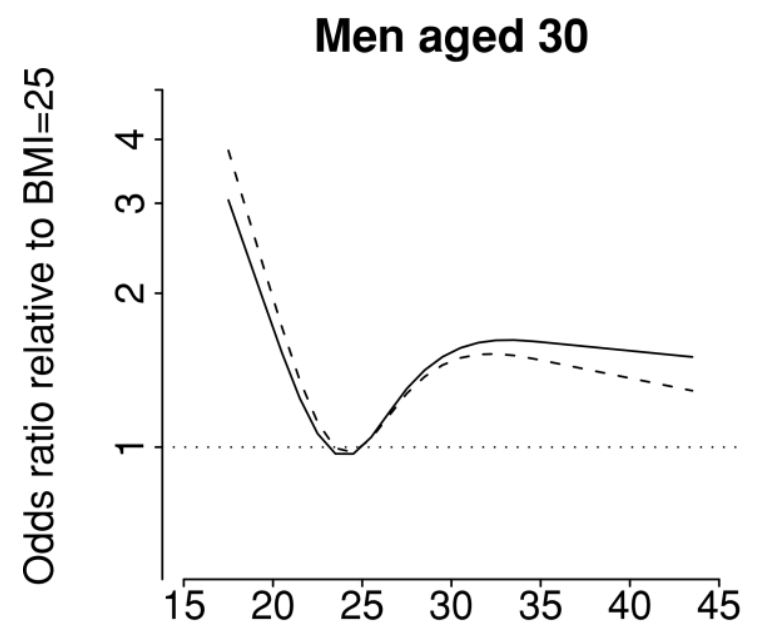

Women aged 30
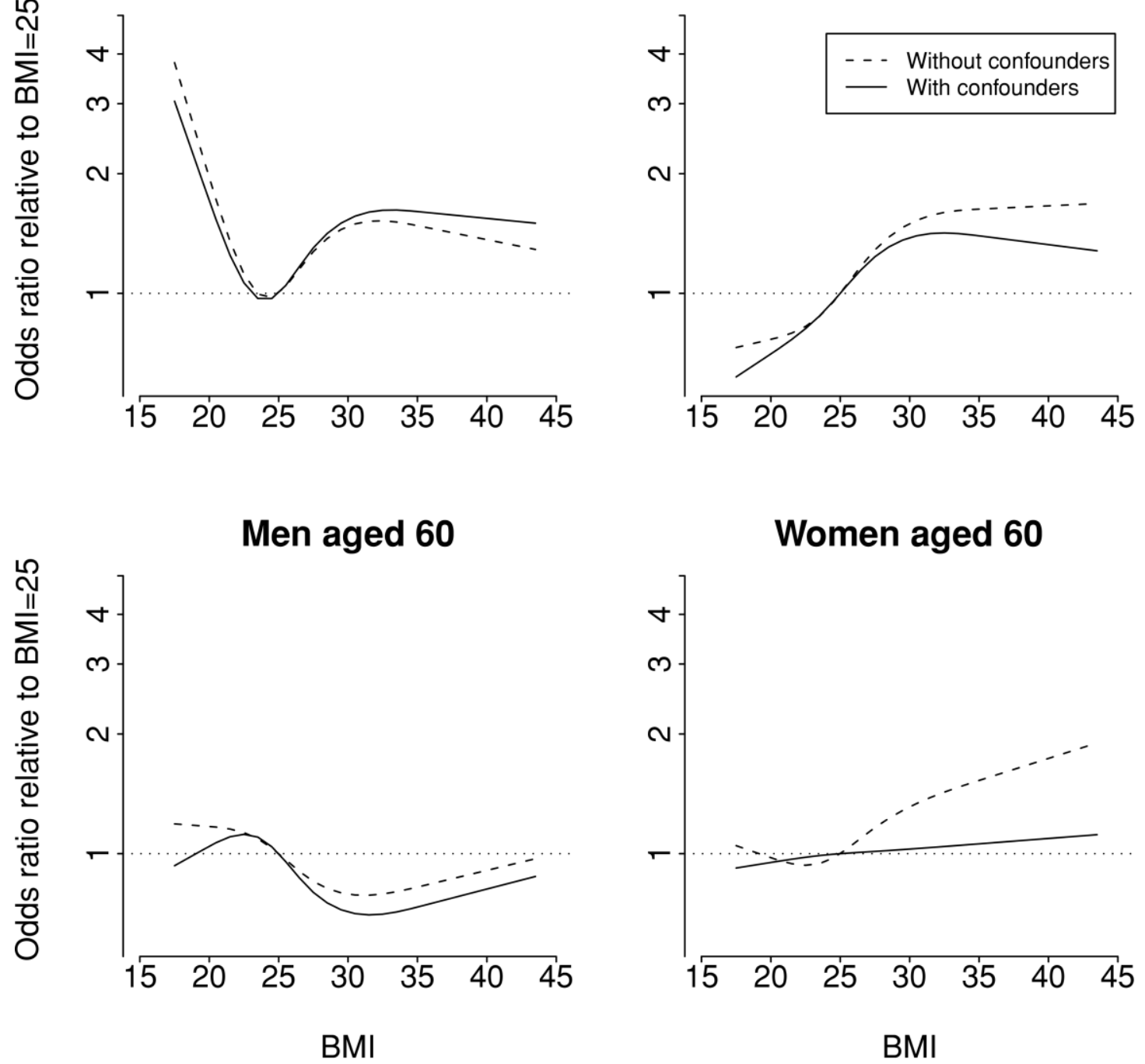

Figure 4: Comparisons of selected odds ratios (relative to a BMI of 25) for the models with and without potential confounders. Odds ratios shown are for individuals with an educational qualification at secondary school level. The vertical axis is plotted on the logistic scale for odds ratios ranging from 0.6 to 5 . 